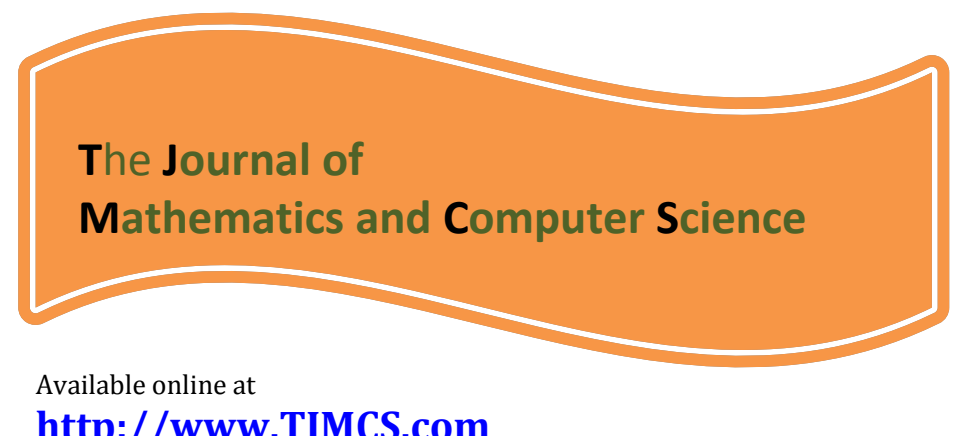

The Journal of Mathematics and Computer Science Vol .3 No.3 (2011) 278 - 286

\title{
Approximate Solution of a Class of Nonlinear Volterra Integral Equations
}

\author{
Hamid Reza Erfanian and Touraj Mostahsan \\ Department of Mathematics \& Statistics, University of Science and Culture, Tehran, Iran. \\ erfanian@ usc.ac.ir and mostahsan@usc.ac.ir
}

Received: May 2011, Revised: November 2011

Online Publication: December 2011

\begin{abstract}
In this paper we introduce an approach by an optimization method to find approximate solution for a class of nonlinear Volterra integral equations of the first and second kind. To this purpose, we consider two stages of approximation. First we convert the integral equation to a moment problem and then we modify the new problem to two classes of optimization problems, non-constraint optimization problems and optimal control problems. Finally numerical examples is proposed.
\end{abstract}

Keywords: Volterra integral equation, Optimal control, Measure theory, Nonlinear and linear programming.

\section{Introduction}

The most of modelling problems of science, engineering, physics and other disciplines leads to linear and nonlinear Volterra integral equations. These are usually difficult to solve analytically and in many cases the solution must be approximated. Therefore, in recent years several numerical approaches have been proposed. The numerical methods usually transform the integral equation into a linear or nonlinear system that can be solved by direct or iterative methods(see [4]).

But it seems that the idea of finding approximate solutions for some problems by converting them to an optimization problem will give rise to interesting results. 
Especially, using measure theory for solving optimal control problems that was established by Rubio [10] gives us a suitable tool to obtain better approximate solutions for this problems. The problem of finding numerical solution for integral equation is one of the oldest problems in applied mathematics and many computational methods are proposed in this area (see [1], [3] and [5]).

We consider a class of nonlinear Volterra integral equations

$$
x(t)=f(t)+\int_{0}^{t} K(s, x(s)) f(s) d s, \quad t \in[0, T] \quad(T<\infty)
$$

where $f:[0, T] \rightarrow \mathbb{R}$ and the kernel $K:[0, T] \times \mathbb{R} \rightarrow \mathbb{R}$ are assumed to be known continuous functions and differentiable and the unknown function to be determined is $x:[0, T] \rightarrow \mathbb{R}$. The necessary and sufficient conditions of existence and uniqueness of the solution for the above problem can be found in [8]. Here, it is assumed that the problem, which is being considered.

The work is structured in three parts: in Section 2, we will convert problem(1) to a optimal control problem. In Section 3, we define the approximating functions and we obtain a finite linear programming. Finally, the numerical results given in Section 4 show the high accuracy of the method.

\section{Transformation}

In this section, we shall present the technique that converts Volterra integral equations (1) to equivalent optimal control problem. This may be achieved by using the Leibnitz rule of differentiating both sides of equation (1) with respect to $t$ and we obtain the equation (see [9])

$$
\dot{x}(t)=\dot{f}(t)+K(t, x(t)) f(t) \quad t \in[0, T] .
$$

Let $\dot{x}(t)=u(t)$ and can be written as

$$
\min \quad I=\int_{0}^{T}|u(t)-\dot{f}(t)-K(t, x(t)) f(t)| d t
$$

subject to

$$
\dot{x}(t)=u(t), \quad t \in[0, T]
$$

Definition 1. We shall say that the pair $w=(x(),. u()$.$) is admissible if the$ following conditions hold:

(i) $u$ (.) is an artificial control function and takes its value in a set $U$.

(ii) $x$ (.) is a differentiable function and takes its values in a set $A$ and we call it trajectory as well.

(iii) The pair $w$ satisfies the differential equation (4) on $(0, T)$.

Let $\Omega=J \times A \times U$ where $J=[0, T]$.We assume that the set of all admissible pairs are nonempty and denoted by $W$.In fact in problems (3) and (4) we are seeking to find the function $u\left(\right.$.) that minimizes the functional $I: W \rightarrow \mathbb{R}^{+}$and satisfies the condition (4).

Now we transform the problem of minimizing (3) with the condition (4) by considering a view of generalization in conditions where we want to convert condition (4) to similar conditions in the objective function (3). Let $B$ be an open 
ball in $\mathbb{R}^{2}$ containing $J \times A$ and $C^{1}(B)$ be the space of all real-valued continuous differentiable functions on it. Let $\varphi \in C^{1}(B)$ and define functions $\varphi^{g}$ as follows:

$$
\varphi^{g}(t, x, u)=\varphi_{x}(t, x) u(t)+\varphi_{t}(t, x) \quad(t, x, u) \in \Omega
$$

The function $\phi^{g}$ is in the space $C(\Omega)$ of all real-valued continuous functions defined on the compact set $\Omega$. Thus we have

$$
\begin{aligned}
\int_{J} \varphi^{g}(t, x, u) d t & =\int_{J}\left(\varphi_{x}(t, x) \dot{x}+\varphi_{t}(t, x)\right) d t \\
& =\int_{J} \dot{\varphi}(t, x) d t=\varphi\left(T, x_{T}\right)-\varphi\left(0, x_{0}\right) \\
& =\Delta \varphi \quad, \forall \varphi \in C^{\prime}(B)
\end{aligned}
$$

for all $\varphi \in C^{1}(B)$, where $\varphi\left(T, x_{T}\right)$ and $\varphi\left(0, x_{0}\right)$ are known.

Let $D\left(J^{0}\right)$ be the space of infinitely differentiable real-valued functions with compact support in $J^{0}$. Define

$$
\psi_{j}(t, x(t), u(t))=x_{j}(t) \psi^{\prime}(t)+u_{j}(t) \psi(t)
$$

for $j=1,2, \ldots, n$ and $\psi \in D\left(J^{0}\right)$, where $u_{j}$ is the $j$ th component of the control function $u$.Then, if $p=(x(),. u()$.$) be an admissible pair , we have for$ $j=1,2, \ldots, n$ and $\psi \in D\left(J^{0}\right)$, $\int_{0}^{T} \psi_{j}(t, x(t), u(t))=\int_{0}^{T} x_{j}(t) \psi^{\prime}(t) d t+\int_{0}^{T} u_{j}(t) \psi(t) d t$ $=\left.x_{j}(t) \psi(t)\right|_{J}-\int_{0}^{T}\left(\dot{x}_{j}(t)-u_{j}(t)\right) \psi(t) d t=0$,

since the function $\psi$ has compact support in $J^{0}, \psi(0)=\psi(T)=0$. With the choice of functions which depend only on the time variable, we have

$\int_{0}^{T} k(t, x(t), u(t)) d t=a_{k}, \quad k \in C_{1}(\Omega)$,

where $C_{1}(\Omega)$ is subspace of the space $C(\Omega)$,of the continuous functions depends only on the time variable $t$. Now consider:

(1) The mapping

$\Lambda_{w}: F \rightarrow \int_{J} F(t, x(t), u(t)) d t, \quad F \in C(\Omega)$, defines a positive linear functional on $C(\Omega)$.

(2) By the Riesz representation theorem (see [10]), there exists a unique positive Radon measure $\mu$ on $\Omega$ such that

$\Lambda_{w}(F)=\int_{J} F(t, x(t), u(t)) d t=\int_{\Omega} F d \mu \equiv \mu(F), \quad F \in C(\Omega)$. 
Thus, the minimization of the functional (3) over constraint (4) is equivalent to the minimization of

$$
I[w]=\Lambda_{w}\left(f_{0}\right)
$$

subject to

$$
\begin{aligned}
& \Lambda_{w}\left(\varphi^{g}\right)=\Delta \varphi, \quad \varphi \in C^{\prime}(B) \\
& \Lambda_{w}\left(\psi_{j}\right)=0, \quad j=1,2, \ldots, n, \psi \in D\left(J^{0}\right) \\
& \Lambda_{w}(k)=a_{k}, \quad k \in C_{1}(\Omega),
\end{aligned}
$$

where $f_{0}=u(t)-\dot{f}(t)-K(t, x(t)) f(t)$.

Now, suppose that the space of all positive Radon measures on $\Omega$ will be denoted

by $M^{+}(\Omega)$. By the Riesz representation theorem, the positive linear functionals above will be replaced by their representing measures, so we seek a measure in $M^{+}(\Omega)$, to be normally denoted by $\mu^{*}$ which minimizes the functional (7).

Thus, the minimization of the functional $I$ in (7) over $W$ is equivalent to the minimization of

$$
I[\mu]=\mu\left(f_{0}\right)
$$

over the set of all positive measures $\mu$ corresponding to admissible pairs $w$, which satisfy

$$
\begin{aligned}
& \mu\left(\varphi^{g}\right)=\Delta \varphi, \quad \varphi \in C^{\prime}(B) \\
& \mu\left(\psi_{j}\right)=0, \quad j=1,2, \ldots, n, \psi \in D\left(J^{0}\right) \\
& \mu(k)=a_{k}, \quad k \in C_{1}(\Omega),
\end{aligned}
$$

We shall consider the minimization of (9) over the set $Q$ of all positive Radon measures on satisfying (10). Now if we topologize the space $M^{+}(\Omega)$ by the weak* topology, it can be seen (see [10]) that $Q$ is compact. The functional $I: Q \rightarrow \mathbb{R}$, defined by

$$
I[\mu]=\int_{\Omega} f_{0} d \mu \equiv \mu\left(f_{0}\right) \in R, \mu \in Q
$$

is a linear continuous functional on a compact set $Q$; so attains its minimum on $Q$ (see [10]), thus, the measure-theoretical problem, which consists of finding the minimum of the functional (9) over the subset $Q$ of $M^{+}(\Omega)$, possesses a minimizing solution $\mu^{*}$, say, in $Q$.

\section{Approximation}

For the estimation by the nearly optimal piecewise constant control, consider the minimization of the functional (9) not over the the set $Q$ but over a subset of $M^{+}(\Omega)$ which is defined by requiring that only a finite number of the constraint in (10) to be satisfied. This will be achieved by choosing countable sets of functions whose linear combinations are dense in the appropriate spaces, and then selecting a finite number of them. In the first step, we obtain an approximation of the optimal measure $\mu^{*}$ by a finite combination of atomic measures, that is, from the Theorem ( see [10], appendix,Theorem A.5), $\mu^{*}$ has 
the form $\mu^{*}=\sum_{i=1}^{N} \alpha_{i}^{*} \delta\left(z_{i}^{*}\right)$ where $\alpha_{i}^{*} \geq 0$ and $z_{i}^{*} \in \Omega$ for $i=1,2, \ldots, N$ (here $\delta(z)$ ) is a unitary atomic measure, characterized by $\delta(z)(F)=F(z)$ where $F \in C(\Omega)$ and $z \in \Omega$ ). Then, we construct a piecewise constant control function corresponding to the finite-dimensional problem ( see [10]). Therefore in the infinite-dimensional linear programming problem (9) with restriction defined by (10), we shall consider only a finite number $M_{1}$ of functions $\varphi$ of the type $\varphi_{1}=x_{1}, \varphi_{2}=x_{2}, \ldots, \varphi_{n}=x_{n}, \varphi_{n+1}=x_{1}^{2}, \varphi_{n+2}=x_{2}^{2}, \ldots$. Also, only a finite number of functions $\chi_{h}, h=1,2, \ldots, M_{2}$ that before defined in (6), when the functions $\psi$ are considered as $\sin (2 \pi r t / T), 1-\cos (2 \pi r t / T), r=1,2, \ldots$, and also, only a finite number $L$ of functions $k$ of the type

$k_{s}(t)= \begin{cases}1 & t \in J_{s} \\ 0 & \text { otherwise }\end{cases}$

will be considered, where $J_{S}=((s-1) d, s d)$, and $d=T / L, s=1,2, \ldots, L$.

The set $\Omega=J \times A \times U$ is covered by a partition, where the partition is defined by taking all points in $\Omega$ as $z_{j}=\left(t_{j}, x_{1_{j}}, x_{2_{j}}, \ldots, x_{n_{j}}, u_{1_{j}}, u_{2_{j}}, \ldots, u_{n_{j}}\right)$. Of course, we only need to construct the control function $u($.), since the trajectory is then simply the corresponding solution of the differential equation (4), with condition $x(0)=x_{0}$, which can be estimated numerically. The infinite-dimensional linear programming problem (9) with restriction defined by (10) can be approximated by the following problem, which $z_{j}$ for $j=1,2, \ldots, N$ belong to an approximately dense subset of $\Omega$.

minimize $\quad \sum_{j=1}^{N} \alpha_{j} f_{0}\left(z_{j}\right)$

subject to

$$
\begin{aligned}
& \sum_{j=1}^{N} \alpha_{j} \varphi_{i}^{g}\left(\mathrm{z}_{j}\right)=\Delta \varphi_{i}, \mathrm{i}=1,2, \ldots, \mathrm{M}_{1} \\
& \sum_{j=1}^{N} \alpha_{j} \chi_{h}\left(\mathrm{z}_{j}\right)=0, \mathrm{~h}=1,2, \ldots, \mathrm{M}_{2} \\
& \sum_{j=1}^{N} \alpha_{j} k_{s}\left(\mathrm{t}_{j}\right)=a_{k}, \mathrm{~s}=1,2, \ldots, \mathrm{L} \\
& \alpha_{j} \geq 0, j=1,2, \ldots, N
\end{aligned}
$$

Note that the elements $z_{j}, j=1,2, \ldots, N$ are fixed, and the only unknowns are the numbers $\alpha_{j}, j=1,2, \ldots, N$.

The procedure to construct a piecewise constant control function approximating the action of the optimal measure is based on the analysis (see [10]).

\section{Numerical examples}

In this section, we propose our method to obtain approximate solution of some Volterra integral equations. 
Example 1. Consider the following nonlinear Volterra integral equation in $[0,1]$ with the analytical solution $x(t)=e^{t}$,

$x(t)=e^{t}-\frac{1}{2}\left(e^{2 t}-1\right)+\int_{0}^{t} x^{2}(s) d s, \quad t \in[0,1]$.

In this example, we consider the set $\Omega=J \times A \times U=[0,1] \times[1, e] \times[1, e]$.We divide the intervals $J, A$ and $U$ into 10 equal subinterval and so $N=10^{3}$.In the next step, we choose the points $z_{j}=\left(t_{j}, x_{j}, u_{j}\right)$ from the set $\Omega$ and select $M_{1}=1, M_{2}=8$ and $L=10$.So we have the following linear programming problem,

$\operatorname{minimize} \quad \sum_{j=1}^{1000}\left|u_{j}-e^{t_{j}}+e^{2 t_{j}}-x_{j}^{2}\right| \alpha_{j}$

subject to $\quad \sum_{j=1}^{1000} u_{j} \alpha_{j}=e-1$

$$
\begin{gathered}
\sum_{j=1}^{1000}\left[2 \pi h x_{j} \cdot \cos \left(2 \pi h t_{j}\right)-u_{j} \sin \left(2 \pi h t_{j}\right)\right] \alpha_{j}=0, \mathrm{~h}=1,2,3,4 . \\
\sum_{j=1}^{1000}\left[2 \pi h x_{j} \cdot \cos \left(2 \pi h t_{j}\right)-u_{j}\left(1-\cos \left(2 \pi h t_{j}\right)\right)\right] \alpha_{j}=0, \mathrm{~h}=5,6,7,8 . \\
\alpha_{1+100(i-1)}+\alpha_{2+100(i-1)}+\ldots+\alpha_{100+100(i-1)}=0.1 \quad, i=1,2, \ldots, 10 . \\
\alpha_{j} \geq 0, j=1,2, \ldots, 1000 .
\end{gathered}
$$

By solving this problem, we obtain $\alpha_{j}, j=1,2, \ldots, 1000$ and then we find the approximate solution $x$ (.). Graph of the approximate solution $\mathrm{x}($.) is shown in Fig.1.

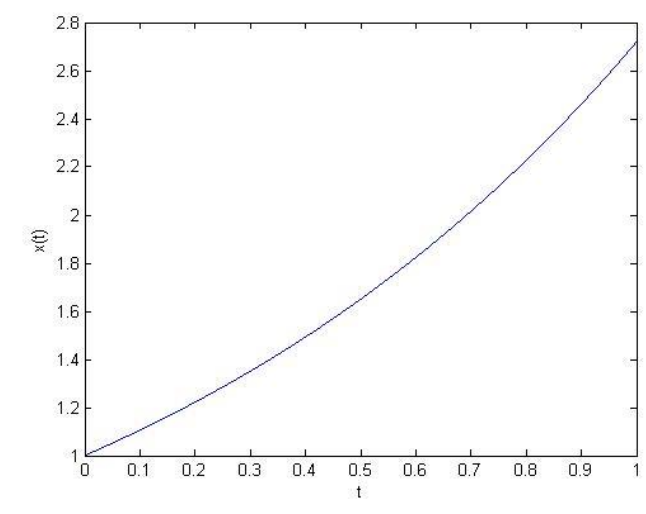

Fig 1. Approximate solution x(.)

Example 2. We apply our method for the following nonlinear Volterra integral equation in $[0,1]$. The analytical solution of the following integral equation is $x(t)=0$. 
$x(t)=\int_{0}^{t} \frac{s x(s)}{1+s+x(s)} d s, \quad t \in[0,1]$.

We consider the set $\Omega=J \times A \times U=[0,1] \times[-1,1] \times[-1,1]$. We divide the intervals $J, A$ and $U$ into 10 equal subinterval and so $N=10^{3}$.In the next step, we choose the points $z_{j}=\left(t_{j}, x_{j}, u_{j}\right)$ from the set $\Omega$ and select $M_{1}=1, M_{2}=8$ and $L=10$.So we have the following linear programming problem,

$$
\operatorname{minimize} \quad \sum_{j=1}^{1000}\left|u_{j}-\frac{t_{j} x_{j}}{1+t_{j}+x_{j}}\right| \alpha_{j}
$$

subject to $\quad \sum_{j=1}^{1000} u_{j} \alpha_{j}=0$

$$
\begin{gathered}
\sum_{j=1}^{1000}\left[2 \pi h x_{j} \cdot \cos \left(2 \pi h t_{j}\right)-u_{j} \sin \left(2 \pi h t_{j}\right)\right] \alpha_{j}=0, \mathrm{~h}=1,2,3,4 . \\
\sum_{j=1}^{1000}\left[2 \pi h x_{j} \cdot \cos \left(2 \pi h t_{j}\right)-u_{j}\left(1-\cos \left(2 \pi h t_{j}\right)\right)\right] \alpha_{j}=0, \mathrm{~h}=5,6,7,8 \\
\alpha_{1+100(i-1)}+\alpha_{2+100(i-1)}+\ldots+\alpha_{100+100(i-1)}=0.1 \quad, i=1,2, \ldots, 10 . \\
\alpha_{j} \geq 0, j=1,2, \ldots, 1000 .
\end{gathered}
$$

Figure 2 represents the result for this example.

Example 3. Consider the following integral equation with the exact solution $x(t)=t$.

$x(t)=\frac{1}{2} t(2+t)-2 t \cdot \operatorname{arctg}(t)+\ln \left(1+t^{2}\right)+\int_{0}^{t}(-x(s)+2 \operatorname{arctg}(x(s))) d s, \quad t \in[0,1], x(0)=0$.

In this example, we consider the set $\Omega=J \times A \times U=[0,1] \times[0,1] \times[0,1]$. The next step is similar to last examples. So we have the following linear programming problem

$\operatorname{minimize} \quad \sum_{j=1}^{1000}\left|u_{j}-\left(1+t_{j}\right)+2 \operatorname{arctg}\left(t_{j}\right)+x_{j}-2 \operatorname{arctg}\left(x_{j}\right)\right| \alpha_{j}$

subject to

$$
\begin{aligned}
& \sum_{j=1}^{1000} u_{j} \alpha_{j}=1 \\
& \sum_{j=1}^{1000}\left[2 \pi h x_{j} \cdot \cos \left(2 \pi h t_{j}\right)-u_{j} \sin \left(2 \pi h t_{j}\right)\right] \alpha_{j}=0, \mathrm{~h}=1,2,3,4 . \\
& \sum_{j=1}^{1000}\left[2 \pi h x_{j} \cdot \cos \left(2 \pi h t_{j}\right)-u_{j}\left(1-\cos \left(2 \pi h t_{j}\right)\right)\right] \alpha_{j}=0, \mathrm{~h}=5,6,7,8 . \\
& \alpha_{1+100(i-1)}+\alpha_{2+100(i-1)}+\ldots+\alpha_{100+100(i-1)}=0.1 \quad, i=1,2, \ldots, 10 . \\
& \alpha_{j} \geq 0, j=1,2, \ldots, 1000 .
\end{aligned}
$$

The approximate solution is shown in Fig.3. 


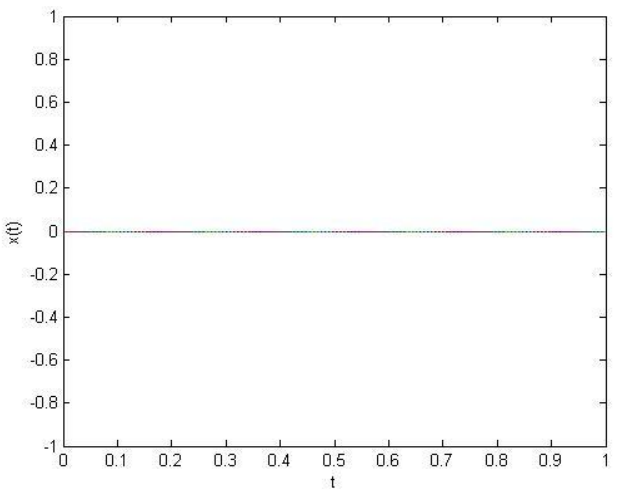

Fig 2. Approximate solution x(.)

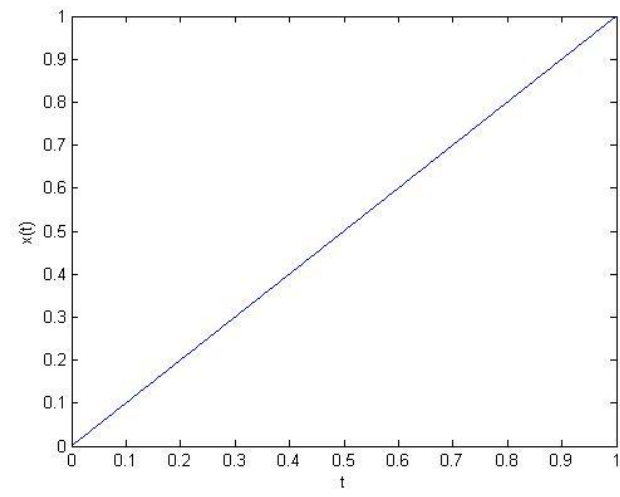

Fig 3. Approximate solution x(.)

\section{Conclusion}

This paper presents a method to find the solution of a class of nonlinear Volterra integral equations by an optimization method that is based on some principles of measure theory, functional analysis and linear programming. In comparison to the other methods, this approach has some advantages. For example, this method is not iterative and it solves the problem directly, without need of any initial guess.

\section{References}

[1] C. T. H. Baker, The Numerical Treatment of Integral Equations, Monographs on Numerical Analysis, Clarendon Press, Oxford, UK, 1977.

[2] C. T. H. Baker, A perspective on the numerical treatment of Volterra equations, Journal of Computational and Applied Mathematics, vol. 125, no. 1-2, pp. 217-249, 2000.

[3] H. Basirzadeh, A. V. Kamyad and S. Effati, AN Approach for Solving Nonlinear

Programming Problems, Korean J. Comput. \& Appl. Math. Vol. 9, No. 2, pp. 547 560, 2002.

[4] M. I. Berenguer, D. Ga'mez, A. I. Garralda-Guillem, and M. C. Serrano P'erez, Nonlinear Volterra Integral Equation of the Second Kind and Biorthogonal Systems,

Abstract and Applied Analysis, Vol. 2010, Article ID 135216, 11 pages.

[5] A. H. Borzabadi , A.V.Kamyad, H.H.Mehne, A different approach for solving the nonlinear Fredholm integral equations of the second kind, Applied Mathematics and Computation, Vol.173, pp.724-735, 2006.

[6] H. Brunner and P. J. van der Houwen, The Numerical Solution of Volterra Equations, vol. 3 of CWI Monographs, North-Holland, Amsterdam, The Netherlands, 1986.

[7] L. M. Delves and J. L. Mohamed, Computational Methods for Integral Equations, Cambridge University Press, Cambridge, Mass, USA, 1985.

[8] A.J.Jerri, Introduction to Integral Equations with Applications, John Wiley and Sons, London , 1999. 
[9] M . Rahman , Integral Equations and their Applications , WIT press, 2007.

[10] J. E. Rubio, Control and Optimization, the Linear Treatment of Non-linear Problems, Manchester University Press, Manchester, UK, 1986.

[11] H. Tian , Spectral Methods for Volterra Integral Equations,a thesis submitted in partial fulfillment of the requirements for the degree of Master of Science in the Department of Mathematics \& Statistics , Simon Fraser University, 1995. 